\author{
ANNALES \\ POLONICI MATHEMATICI \\ XXXII (1876)
}

\title{
Classification des densités vectorielles factorisées
}

\author{
par EdWand SrwEK (Katowice)
}

Résumé. Soit $\nabla_{t}$ (resp. $W_{t}$ ) la densité vectorielle ordinaire (resp. du type de H. Weyl) de poids $-t \in R$. Comme $V_{t}\left(W_{t}\right)$ sont des objets géomótriques transitifs, toute congruence $C$ pour $\nabla_{t}\left(W_{t}\right)$ prend la forme: $x O x^{\prime} \Leftrightarrow \exists_{A \text { eQ } Q L(n)}\left(x, x^{\prime} \in A G \cdot x_{0}\right)$, où $G$ est un sous-groupe fermé arbitraire de $G L(n)$, contenant le sous-groupe de stabilité de $x_{0}$ par rapport à $\nabla_{\ell}\left(W_{t}\right)$.

Posons pour $A=\left[a_{i j}\right]_{i, j=1, \ldots, n} \in G L(n)$ :

$$
H=\left\{A \in G L(n): a_{21}=\ldots=a_{n 1}=0\right\}, \quad a=a_{11}, d=\operatorname{det}\left[a_{i j}\right]_{i, j=2, \ldots, n}
$$

et définissons les sous-groupes de $H$ : ,

$$
\begin{gathered}
G^{+}=\{A: d>0\}, \quad H^{+}=\{A: a>0\}, \\
G_{0}(c)=\left\{A:|a|=c^{k}, k \in Z\right\}, \quad H_{1}(c)=\left\{A:|d|=c^{k}, k \in Z\right\}, \\
G_{0}^{*}(c)=\left\{A:|a|=c^{k}, \operatorname{sgn} d=(-1)^{k}, k \in Z\right\}, \\
H_{1}^{*}(c)=\left\{A:|d|=c^{k}, \operatorname{sgn} a=(-1)^{k}, k \in Z\right\}, \\
G_{0}^{+}(c)=\left\{A: d>0,|a|=c^{k}, k \in Z\right\}, \\
H_{1}^{+}(c)=\left\{A: a>0,|d|=c^{k}, k \in Z\right\},
\end{gathered}
$$

et pour $t \neq 0$ resp. $t \neq 1$ :

$$
\begin{aligned}
G_{t}(c) & =\left\{A:|d|=c^{k}|a|^{(1-t) / l}, k \in Z\right\}, \\
H_{t}(c) & =\left\{A:|a|=c^{k}|d|^{t /(1-t)}, k \in Z\right\}, \\
G_{t}^{*}(c) & =\left\{A: d=(-c)^{k}|a|^{(1-t) / t}, k \in Z\right\}, \\
H_{t}^{*}(c) & =\left\{A: a=(-c)^{k}|d|^{t /(1-t)}, k \in Z\right\}, \\
G_{t}^{+}(c) & =\left\{A: d=c^{k}|a|^{(1-t) / t}, k \in Z\right\}, \\
H_{t}^{+}(c) & =\left\{A: a=c^{k}|d|^{t /(1-t)}, k \in Z\right\},
\end{aligned}
$$

où $\boldsymbol{Z}$ est l'ensemble des entiers. On a obtenu le résultat suivant:

Toute factorisation (comitant algébrique) de la densité $V_{t}\left(W_{t}\right)$ est équivalente à l'un des objets factorisés suivants: $\Sigma=V_{l} / G L(n)$ (scalaire trivial), $\Delta=\nabla_{\mathbb{l}} / H$ (direction), $\Delta^{*}=V_{t} / G^{+}, \nabla_{t}(c)=\nabla_{t} / G_{l}(c)$ pour $c>1, V_{t}^{*}(c)=V_{t} / G_{t}^{*}(c)$ pour $c>1$ et $\nabla_{t}^{+}(c)=V_{t} / G_{t}^{+}(c)$ pour $c \geqslant 1$ [resp. $\Sigma=W_{t} / G L(n)$ (scalaire trivial), $\Delta=W_{t} / H$ (direction), $\Delta^{+}=W_{l} / H^{+}$(direction munie d'un sens), $W_{l}(c)=W_{l} / H_{l}(c)$ pour $c>1$, $W_{t}^{*}(c)=W_{t} / H_{i}^{*}(c)$ pour $c>1, W_{t}^{+}(c)=W_{t} / H_{t}^{+}(c)$ pour $c>1$ ]. Les objets nommés ci-dessus ne sont pas équivalents l'un à l'autre.

Introduction. Dans la théorie des objets géométriques on considère le problème de la classification des comitants algébriques des objets 
géométriques abstraits( $\left.{ }^{1}\right)$ d'un type fixe. Ce problème consiste à déterminer, jusqu'à l'équivalence, tous les comitants d'un objet géométrique abstrait du type donné. Pourtant on simplifie souvent ce problème en fixant non seulement le type de l'objet géométrique considéré, mais aussi le type des comitants cherchés.

En remarquant que le problème de la classification des comitants est équivalent à celui de la classification des objets géométriques factorisés (voir [6], théorèmes 1 et 2) on peut éviter des simplifications supplémentaires. En utilisant cette remarque, ainsi qu'un théorème bien connu caractérisant les sous-groupes fermés du groupe multiplicatif des nombres réels on a donné dans la note [7] une classification complète des comitants (et aussi des objets géométriques factorisés) pour des densités. Le but de la présente note est d'étendre cette méthode aux densités vectorielles, lesquelles peuvent aussi être envisagées comme des objects géométriques transitifs.

1. Factorisation des objets géométriques abstraits. Un objet géométrique abstrait de la classe $r$ peut être envisagé comme une structure $\boldsymbol{X}$ $=(G, X, \cdot)$, composée d'un sous-groupe topologique $G$ du groupe différentiel d'ordre $r$, d'un espace topologique $X$ appelé fibre de l'objet géométrique $\boldsymbol{X}$ et d'une opération continue ," ":

$$
G \times X^{3}(g, x) \mapsto g \cdot x \in X
$$

constituant une représentation du groupe $G$ dans le groupe Hom $X$ des homéomorphismes de l'espace $X$ sur lui-même. Il en résulte que l'opération ," satisfait, pour $\infty \in X$ et $g_{1}, g_{2} \in G$, aux conditions suivantes:

$$
g_{2} \cdot\left(g_{1} \cdot x\right)=\left(g_{2} g_{1}\right) \cdot x, \quad e \cdot x=w,
$$

où $e$ désigne l'unité du groupe $G$. On appelle l'opération ,", loi de transformation de l'objet géométrique $\boldsymbol{X}$. Dans le cas où le groupe $G$ opère par ,," de façon transitive sur $X$, l'objet géométrique $\boldsymbol{X}$ est dit transitif. Un objet géométrique $\boldsymbol{Y}=(G, Y, *)$ sera dit comitant de l'objet géométrique $\boldsymbol{X}$ si la structure $(G, Y, *)$ est homomorphe à celle $(G, X, \cdot)$, c'est-à-dire s'il existe une application continue $\varphi$ de $X$ sur $Y$ et satisfaisant, pour $g \in G$ et $\boldsymbol{x} \epsilon \boldsymbol{X}$, à la condition suivante:

$$
\varphi(g \cdot \boldsymbol{\infty})=g * \varphi(\boldsymbol{\infty}) .
$$

Dans le cas où les structures $(G, X, \cdot)$ et $(G, Y, *)$ sont isomorphes on dit que les objets correspondants $\boldsymbol{X}$ et $\boldsymbol{Y}$ sont équivalents.

Soit $C$ une congruence pour l'objet géométrique $X$, c'est-à-dire une relation fermée, réflexive, symétrique et transitive dans $X$ et satisfaisant

(1) Pour les notions de la théorie des objets géométriques qui ne sont pas expliquées ici voir p. ex. [3] ou [6]. 
à la condition suivante:

$$
\left(w, w^{\prime}\right) \in C \Rightarrow\left(g \cdot w, g \cdot w^{\prime}\right) \in C
$$

pour $g \in G$. En posant:

$$
g \cdot[w]=[g \cdot \infty],
$$

où $g \in G, \infty \in X$ et $[\omega]=\left\{w^{\prime} \in X:\left(\infty, x^{\prime}\right) \in C\right\}$ l'opération ," détermine la loi de transformation de l'objet géométrique suivant:

$$
X / C=(G, X / C, \cdot)
$$

considéré comme une factorisation de l'objet $X$ par rapport à la congruence $C$. Fixons $\infty_{0} \in X$ et posons:

$$
S_{0}=\left\{g \in G: g \cdot \infty_{0}=\infty_{0}\right\} .
$$

Il en résulte facilement, que pour tout $\varpi_{0} \in X, \mathbb{S}_{0}$ est un sous-groupe fermé de $G$, appelé sous-groupe de stabilité du point $x_{0}$. On constate aussi que les sous-groupes de stabilité qui correspondent à des points différents de la fibre $X$ d'un objet géométrique transitif $X$ sont conjugués l'un de l'autre. Tout sous-groupe fermé $S$ de $G$ contenant le sous-groupe $S_{0}$ de stabilité d'un point fixe $x_{0} \in X$ détermine une congruence $C_{S}$ par la formule

$$
\left(x, x^{\prime}\right) \in C_{S} \Leftrightarrow \exists_{\sigma \leftrightarrow G}\left(x, x^{\prime} \in g S \cdot x_{0}\right)
$$

et, par conséquent, le sous-groupe $\$ \mathbb{S}$ détermine un objet géométrique factorisé $X / C_{S}$ noté dans la suite $X / S$.

Dans la note [6] nous avons établi la proposition suivante:

Proposition 1. Soit $\boldsymbol{X}$ un objet géométrique transitif et $C$ une congruence pour $\boldsymbol{X}$. Tout objet géométrique factorisé de la forme $\boldsymbol{X} / C$ est équivalent $\grave{a}$ un objet de la forme $\mathbf{X} / S$, où $S$ désigne un sous-groupe fermé du groupe $G$ contenant un sous-groupe $S_{0}$ de stabilité d'un point fixe $x_{0} \in X$.

Comme tout objet géométrique factorisé de la forme $X / \mathcal{S}$ est aussi de la forme $\boldsymbol{X} / C$, il en résulte que le problème général de la classification des objets factorisés se réduit à celui des factorisations par rapport aux sous-groupes fermés contenant un sous-groupe de stabilité d'un point fixe. Pour effectuer cette classification nous utiliserons la proposition suivante:

Proposition 2. Soient $\boldsymbol{X}=(G, X, \cdot)$ un objet géométrique transitif, $S$ et $\tilde{S}$ - des sous-groupes fermés du groupe $G$ tels que l'on ait: $S \supset S_{0}$ et $\tilde{\mathbb{S}} \supset \overline{\mathbb{S}}_{0}$, ou $\mathbb{S}_{0}$ resp. $\tilde{\mathbb{S}}_{0}$ designe le sous-groupe de stabilité d'un point $x_{0} \in X$ resp. $\tilde{x}_{0} \in X$. Pour que les objets géométriques factorisés $\boldsymbol{X} / S$ et $\boldsymbol{X} / \tilde{S}$ soient équivalents il faut et il suffit que les sous-groupes $S$ et $\tilde{S}$ soient conjugués.

Démonstration. Nécessité. Désignons par [x] resp. 〈ळ〉 un élément de la fibre $X / S$ resp. $X / \tilde{S}$ satisfaisant à la condition:

$$
\boldsymbol{x} \in[\boldsymbol{x}], \quad \text { resp. } \boldsymbol{x} \in\langle\boldsymbol{x}\rangle \text {. }
$$


En vertu de la transitivité de $X$ et de la définition (2) pour tout $\varpi \in X$ il existe des $g_{x} \in G$ et $\tilde{g}_{x} \in G$ tels que l'on ait:

$$
[\omega]=g_{x} \cdot\left[\omega_{0}\right]=g_{x} S \cdot \omega_{0} \quad \text { et } \quad\langle\omega\rangle=\tilde{g}_{x} \cdot\left\langle\tilde{x}_{0}\right\rangle=\tilde{g}_{x} \tilde{\mathcal{S}} \cdot \tilde{x}_{0} .
$$

D'après l'équivalence des objets factorisés $X / S$ et $X / \tilde{S}$ il existe un homéomorphisme $\varphi$ de $X / S$ sur $X / \tilde{S}$ satisfaisant à la condition de la forme (1). II en résulte l'existence de $h \in G$ tel que l'application $\varphi$ se laisse définir par les conditions suivantes:

$$
\varphi\left(\left[\infty_{0}\right]\right)=h \cdot\left\langle\tilde{x}_{0}\right\rangle, \quad \varphi\left(g \cdot\left[\infty_{0}\right]\right)=g \cdot \varphi\left(\left[\omega_{0}\right]\right)=g h \cdot\left\langle\tilde{x}_{0}\right\rangle .
$$

Comme nous avons:

et

$$
g_{1} \cdot\left[\infty_{0}\right] \neq g_{2} \cdot\left[\infty_{0}\right] \Leftrightarrow g_{1} S \cdot w_{0} \neq g_{2} S \cdot w_{0} \Leftrightarrow g_{2}^{-1} g_{1} \& S
$$

$$
\begin{aligned}
& \varphi\left(g_{1} \cdot\left[\omega_{0}\right]\right) \neq \varphi\left(g_{2} \cdot\left[x_{0}\right]\right) \Leftrightarrow g_{1} h \cdot\left\langle\tilde{x}_{0}\right\rangle \neq g_{2} h \cdot\left\langle\tilde{x}_{0}\right\rangle \\
& \Leftrightarrow g_{1} h \tilde{\mathcal{S}} \cdot \tilde{x}_{0} \neq g_{2} h \tilde{\mathcal{S}} \cdot \tilde{x}_{0} \leftrightarrow h^{-1} g_{2}^{-1} g_{1} h \not \tilde{\mathbb{S}}
\end{aligned}
$$

a bijectivité de l'application $\varphi: X / \mathbb{S} \rightarrow X / \tilde{\mathbb{S}}$ entraíne la relation:

$$
h^{-1} g_{2}^{-1} g_{1} h \in \tilde{\mathbb{S}} \Leftrightarrow g_{2}^{-1} g_{1} \in S \quad \text { pour } g_{1}, g_{2} \in G \text {. }
$$

Donc les sous-groupes $\mathcal{S}$ et $\tilde{\mathcal{S}}$ sont conjugués et la nécessité sê trouve démontrée.

Suffisance. Soit $S=h \tilde{S} h^{-1}$ pour un $h \in G$. Alors l'application $\varphi$ : $X / \mathbb{S} \rightarrow X / \tilde{S}$ défine par les conditions (3) doit être bijective. Comme les sous-groupes $S$ et $\tilde{S}$ de $G$ sont fermés, la continuité de $\varphi$ et $\varphi^{-1}$ suit immédiatement de la définition (3) et de la continuité de l'operation $(g, \infty) \mapsto g \cdot \infty$. Donc les objets factorisés $X / \mathscr{S}$ et $X / \tilde{S}$ sont équivalents et la proposition se trouve démontrée.

D'après les proposition 1 et 2 le problème de la classification des comitants d'un objet géométrique transitif, étant équivalent ( ${ }^{2}$ ) à celui de la classification des objets factorisés de la forme $X / S$, se réduit au problème de déterminer tous les sous-groupes fermés de $G$, contenant le sous-groupe $S_{0}$ de' stabilité d'un point $x_{0} \in X$ et n'étant pas conjugués l'un de l'autre. Le choix du point $x_{0}$ ne joue ici aucun rôle et, par conséquent, on peut le choisir d'une manière simplifiant la détermination des sous-groupes $S$.

Dans le cas où $\boldsymbol{X}$ est un objet géométrique transitif toute factorisation de la forme $X / S$ est aussi un objet transitif, $S$ étant le sous-groupe de stabilité de l'élément $\left[x_{0}\right]=S \cdot x_{0}$. Donc, en vertu de la proposition 1 , l'objet $X / S$ n'admet que des factorisations équivalent aux objets de la

(2) Dans le cas où l'objet géométrique donné n'est pas transitif, les problèmes ne sont pas équivalents. Sur ce sujet voir [5]. 
forme $(X / S) / T$, où $T$ est un sous-groupe fermé de $G$ contenant le sous-groupe $S$. Comme $(X / \mathbb{S}) / T$ doit être équivalent à un objet de la forme $X / T$, nous obtenons la proposition suivante:

Proposition 3. Dans le cas à̀ $\boldsymbol{X}$ est un objet géométrique transitif toute factorisation (comitant algébrique) d'un objet de la forme $\mathbf{X} / \mathbb{S}$ est équivalente à un objet de la forme $X / T$, où $T$ est un sous-groupe fermé de $G$ contenant le sous-groupe $S$.

2. Théorèmes auxiliaires. La classification des objets géométriques factorisés correspondant aux densités vectorielles sera basée sur certains théorèmes concernant les sous-groupes fermés de certains groupes. Nous allons présenter ces théorèmes.

ThÉoRìme 1. Le groupe multiplicatif des nombres réels $\boldsymbol{R}_{0}$ ne contient (sauf lui-même) de sous-groupes fermés que les suivants:

$$
\begin{aligned}
\boldsymbol{R}^{+} & =\left\{\varrho \in \boldsymbol{R}_{0}: \varrho>0\right\}, & & \\
\boldsymbol{R}^{+}(c) & =\left\{\varrho \in \boldsymbol{R}_{0}: \varrho=c^{k}, k \in \boldsymbol{Z}\right\} & & \text { pour } c \geqslant 1, \\
\boldsymbol{R}(c) & =\left\{\varrho \in \boldsymbol{R}_{0}:|\varrho|=c^{k}, k \in \boldsymbol{Z}\right\} & & \text { pour } c \geqslant 1, \\
\boldsymbol{R}^{*}(c) & =\left\{\varrho \in \boldsymbol{R}_{0}: \varrho=(-c)^{k}, k \in \boldsymbol{Z}\right\} & & \text { pour } c>1,
\end{aligned}
$$

où $\boldsymbol{Z}$ désigne l'ensemble de tous les nombres entiers $\left(^{8}\right)$.

Admettons quelques notations. Désignons par $H$ l'ensemble des matrices de la forme:

$$
A=\left[a_{j}^{i}\right]_{i, j-1, \ldots,}
$$

satisfaisant aux conditions suivantes:

$$
a_{1}^{2}=\ldots=a_{1}^{n}=0, \quad \operatorname{det} A \neq 0 .
$$

Pour tout $t$ réel désignons encore par $G_{\ell}$ resp. $H_{\imath}$ l'ensemble des matrices de la forme (4) telles que l'on ait:

$$
a_{1}^{1}=|J|^{t} \operatorname{sgn} J, \quad a_{1}^{2}=\ldots=a_{1}^{n}=0, \quad J=\operatorname{det} A \neq 0
$$

resp.

$$
a_{1}^{1}=|J|^{\ell}, \quad a_{1}^{2}=\ldots=a_{1}^{n}=0, \quad J=\operatorname{det} A \neq 0 .
$$

$\boldsymbol{n}$ est facile de vérifier que $H, G_{t}$ et $H_{t}$ forment des sous-groupes fermés du groupe $G L(n)$ pour tout $t$ réel.

Maintenant nous établirons quelques lemmes

LEMME 1. Supposons que:

$G^{\prime}$ soit un sous-groupe du groupe $G L(n)$,

$G_{t} \subset G^{\prime}$ resp. $H_{\imath} \subset G^{\prime}$ pour $t$ réel fixe,

$G^{\prime} \cup H=G L(n)$.

$\left.{ }^{3}\right)$ Ce théorème bien connu a été quelquefois appliqué dans la théorie des objets géométriques (voir p. ex. [2]). Pour la démonstration voir [7]. 
Alors:

$$
G^{\prime}=G L(n) .
$$

Démonstration $\left({ }^{4}\right)$. En vertu des hypothèses il suffit de prouver la relation

$$
H \subset G^{\prime} \text {. }
$$

A e $H$ étant de la forme (4) nous posons:

et

$$
\varepsilon=\operatorname{sgn}\left(\operatorname{det}\left[a_{j}^{i}\right]_{i, j-2, \ldots, n}\right),
$$

$$
A_{i}=\left[\begin{array}{cccc}
|J|^{t} \operatorname{sgn} J & \frac{1}{\varrho} a_{2}^{1} & \ldots & \frac{1}{\varrho} a_{n}^{1} \\
0 & \varepsilon a_{2}^{2} & \ldots & \varepsilon a_{n}^{2} \\
0 & a_{2}^{3} & \ldots & a_{n}^{3} \\
\ldots & \ldots & \ldots & \\
0 & a_{2}^{n} & \ldots & a_{n}^{n}
\end{array}\right], \quad C_{e}=\left[\begin{array}{llll}
\varrho & 0 & & \\
\varrho & \varepsilon & & \\
& & 1 & \\
& & \ddots & \\
& & & 1
\end{array}\right]
$$

où $J=\operatorname{det} A_{t}$, et $\varrho=a_{1}^{1}|J|^{-t} \operatorname{sgn} J$. Alors on vérifie facilement la relation:

$$
A=C_{e} \cdot A_{i}
$$

En utilisant les hypothèses on constate aussi que

$$
C_{e} \in G^{\prime} \quad \text { et } \quad A_{t} \in G^{\prime}
$$

(car $A_{l} \in G_{\ell}$ ). Comme $G^{\prime}$ est un sous-groupe de $G L(n)$ il en résulte

$$
A \in G^{\prime}
$$

donc aussi - l'inclusion (5). Notre lemme se trouve ainsi démontré.

LEMME 2. Tout sous-groupe du groupe $G L(n)$ (sauf lui-même), contenant le sous-groupe $G_{t}$ resp. $H_{t}$ pour $t$ réel fixe, doit étre contenu dans le sous-groupe $\boldsymbol{H}$.

Démonstration $\left({ }^{4}\right)$ Pour la démonstration par le contraire supposons qu'il existe un sous-groupe $\theta^{\prime}$ du $G L(n)$ tel que l'on ait

$$
G_{t} \subset G^{\prime} \neq H \text {. }
$$

Alors il suffit de montrer l'égalité:

$$
G^{\prime}=G L(n)
$$

(4) Nous ne présenterons ici que la démonstration dans le cas des sous-groupes de la forme $G_{l}$. Dans le cas des sous-groupes de la forme $H_{\ell}$ la démonstration n'exige que de petites modifications concernant le choix des matrices auxiliaires. 
De l'hypothese (6) résulte l'existence d'une matrice $A$ telle que l'on a:

$$
G^{\prime} \ni A \notin H
$$

On a aussi:

$$
B=\left[\begin{array}{lllll}
|b|^{-t} \operatorname{sgn} b & & & 0 & \\
& |b|^{t-1} & & \\
& & 1 & & \\
& 0 & & \ddots & 1
\end{array}\right] \epsilon G^{\prime}
$$

pour tout $b \neq 0$ (car $B \in G_{t}$ ). En posant $b=\operatorname{det} A$ il s'ensuit

$$
H \boldsymbol{B} \cdot A \in G^{\prime} \cap S L(n),
$$

où $S L(n)$ désigne le sous-groupe unimodulaire de $G L(n)$. Donc on a aussi:

$$
H \cap S L(n) \subset\left(H \cup G^{\prime}\right) \cap S L(n) \neq H \cap S L(n) .
$$

D'autre part, le groupe $H \cap S L(n)$ - étant composé de toutes les matrices du groupe $\boldsymbol{S L}(n)$ conservant un sous-espace linéaire de $\boldsymbol{R}^{n}-$ est un sous-groupe maximal dans $S L(n)$ (voir [1]). D'après (7) il en résulte la relation:

done aussi

$$
\left(H \cup G^{\prime}\right) \cap S L(n)=\$ L(n)
$$

$$
S L(n) \subset H \cup G^{\prime}
$$

Comme le groupe $H$, donc aussi $H \cup G^{\prime}$, contient toutes les matrices de la forme:

$$
\left[\begin{array}{lll}
\lambda & & \\
& \ddots & \lambda
\end{array}\right],
$$

où $\lambda \neq 0$, la dernière inclusion entraîne l'égalité:

$$
H \cup G^{\prime}=G L(n) \text {. }
$$

Ainsi les hypothèses du lemme 1 sont remplies. Le lemme 1 achève la démonstration.

LEMME 3. Soit $G$ un groupe topologique admettant la forme $G=F \cdot Q$, oì $F$ et $Q$ sont des sous-groupes fermés de $G$. Tout sous-groupe fermé $G^{\prime}$, de $G$, contenant le sous-groupe $Q$, est de la forme: $G^{\prime}=F^{\prime} \cdot Q$, ò̀ $F^{\prime}$ est un sous-groupe fermé de $F$.

Démonstration. D'abord nous montrerons l'implication suivante:

$$
f_{0} \in F, \quad q_{0} \in Q, \quad f_{0} q_{0} \in G^{\prime} \Rightarrow f_{0} Q \subset G^{\prime} .
$$

5 - Annales Polonicl Mathematicl XXxII.s 
En effet, pour $f_{0}$ et $q_{0}$ satisfaisant aux conditions: $f_{0} \in F, q_{0} \in Q, f_{0} q_{0} \epsilon G^{\prime}$ et pour tout $q \in Q$ on $\mathrm{a}$ :

$$
f_{0} q=\left(f_{0} q_{0}\right)\left(q_{0}^{-1} q\right) \epsilon G^{\prime}
$$

parce que $q_{0}^{-1} q \in Q \subset G^{\prime}$. Il en résulte immédiatement $f_{0} Q \subset G^{\prime}$ et l'implication (8) se trouve ainsi démontrée.

Posons maintenant:

$$
F^{\prime}=\left\{f \epsilon F: f Q \subset G^{\prime}\right\}
$$

$\Pi$ en résulte immédiatement l'inclusion:

$$
F^{\prime} Q \subset G^{\prime}
$$

D'autre part, d'après les hypothèses, tout élément de $G^{\prime}$ admet une représentation sous la forme $f q$, où $f \in F$ et $q \in Q$. D'après (8) il en résulte l'inclusion inverse:

$$
G^{\prime} \subset F^{\prime} Q
$$

et, par conséquent, nous obtenons:

$$
G^{\prime}=F^{\prime} Q
$$

Soit maintenant $f_{3}=f_{1} f_{2}$, où $f_{1}, f_{2} \in F^{\prime}$. Alors nous avons:

$$
f_{i}=f_{i} e \epsilon G^{\prime} \quad \text { pour } i=1,2
$$

( $e$ désigne l'unité du groupe $G$ ) et, par conséquent, on a: $f_{3} \in G^{\prime}$ et $f_{3} \in F$. En vertu de (9) et (10) cela implique $f_{3} \in F^{\prime}$, done $F^{\prime}$ forme un sous-groupe de $G^{\prime}$, et aussi de $G$.

Enfin soit

$$
f_{0}=\lim _{v \rightarrow \infty} f_{v},
$$

où $f_{v} \in F^{\prime}$ pour $v \in N$. Alors on a aussi:

$$
f_{,} \in G^{\prime} \quad \operatorname{pour} v \in N
$$

et, en vertu de nos hypothèses, on obtient:

$$
f_{0} \in G^{\prime} \quad \text { et } f_{0} \in F \text {. }
$$

Oomme plus haut il s'ensuit $f_{0} \epsilon F^{\prime}$. Donc $F^{\prime}$ est un sous-groupe ferm $\Leftrightarrow$ de $F$ et le lemme se trouve ainsi démontré.

Enfin nous démontrerons le lemme suivant:

LEMME 4. Dans le cas où un groupe abstrait $\theta$ admet un sous-groupe invariant $Q$ et un sous-groupe $F$ tels que l'on ait:

$$
\boldsymbol{G}=\boldsymbol{F} \boldsymbol{Q}
$$

et

$$
f_{1}, f_{2} \in F \Rightarrow f_{1} f_{2}=f_{2} f_{1}
$$


tout sous-groupe $G^{\prime}$ de $G$, contenant le sous-groupe $Q$, est un sous-groupe invariant de $G$.

Démonstration. Soit $G^{\prime}$ un sous-groupe de $G$ contenant $Q$ et $G^{\prime \prime}$ $=g^{-1} G^{\prime} g$, où $g \in G$. En vertu de (11) on a $G^{\prime}=F^{\prime} Q$, où $F^{\prime} \subset F$.

Comme $Q$ est un sous-groupe invariant de $G$ on a aussi:

$$
G^{\prime \prime}=\left(g^{-1} F^{\prime} g\right)\left(g^{-1} Q g\right)=\left(g^{-1} F^{\prime} g\right) Q
$$

pour tout $g \in G$. En vertu de (11) tout élément $g \in G$ se laisse présenter sous la forme $g=f q$, où $f \in F$ et $q \in Q$. Alors, en vertu de (13) et (12), nous obtenons :

$$
\begin{aligned}
G^{\prime \prime} & =\left(q^{-1} f^{-1} F^{\prime} f q\right) Q=q^{-1}\left(f^{-1} F^{\prime} f\right) Q=q^{-1}\left(F^{\prime} f^{-1} f\right) Q \\
& =q^{-1} F^{\prime} Q=q^{-1} G^{\prime}=G^{\prime}
\end{aligned}
$$

et le lemme se trouve démontré.

3. Application aux densités vectorielles. Pour tout $t \in \boldsymbol{R}$ on appelle densité vectorielle ordinaire de poids $-t$ resp. densité vectorielle du type de $H$. Weyl de poids $-t$ l'objet géométrique abstrait $\nabla_{t}$ resp. $W_{t}$ relatif au groupe $G L(n)$ avec la fibre:

$$
X=\boldsymbol{R}^{n} \backslash\{(0, \ldots, 0)\}
$$

et la loi de transformation défine par la formule:

$$
G L(n) \times \bar{X}^{3}(A, x) \mapsto|J|^{\ell} \operatorname{sgn} J A \cdot x
$$

resp.

$$
G L(n) \times X \ni(A, \infty) \mapsto|J|^{t} A \cdot x,
$$

où $J=\operatorname{det} A$ et , ," désigne la multiplication matricielle. On vérifie facilement la transitivité des objets $V_{t}$ et $W_{t}$ pour $t \in \boldsymbol{R}$. On sait aussi (voir [4]) que les objets $V_{t}$ et $W_{t}$ pour $t \epsilon \boldsymbol{R}$ ne sont pas équivalents l'un à l'autre.

Fixons $x_{0}=(1,0, \ldots, 0) \in X$. Il est facile de vérifier que le sous-groupe de stabilité de l'élément $x_{0}$ par rapport à la densité vectorielle $V_{t}$ resp. $W_{t}$ est le sous-groupe $G_{t}$ resp. $H_{t}$, défini au section 2 . En vertu des propositions 1 et 2 le problème de la classification des densités vectorielles factorisées se réduit à celui de déterminer tous les sous-groupes fermés du groupe $G L(n)$, contenant le sous-groupe $G_{t}$ ou $H_{t}$ (pour tout $t$ réel fixe) et n'étant pas conjugués l'un de l'autre. Mais, en vertu du lemme 2 , le dernier problème se reduit au problème correspondant pour les sous-groupes fermés du groupe $H$, défini au section 2. 
Admettons maintenant les notations suivantes

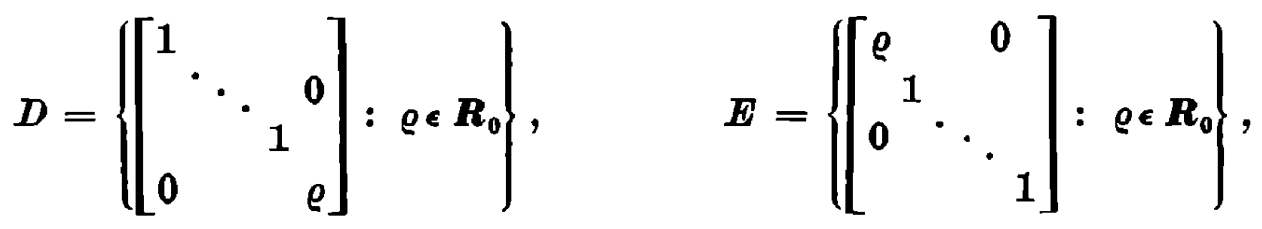

$$
\begin{aligned}
& \left.\boldsymbol{K}=\left\{\left[\begin{array}{llll}
|\varrho| & & & 0 \\
& 1 & & \\
& & \ddots & \\
0 & & & \text { sgn } \varrho
\end{array}\right]: \varrho \in \boldsymbol{R}_{0}\right\}, L=\left\{\begin{array}{cccc}
\operatorname{sgn} \varrho & & & 0 \\
& 1 & & \\
& & \ddots & 1 \\
0 & & & |\varrho|
\end{array}\right]: \varrho \in \boldsymbol{R}_{0}\right\}
\end{aligned}
$$

et posons pour $A$ de la forme (4):

$$
a(A)=a_{1}^{1} \quad \text { et } \quad d(A)=\operatorname{det}\left[a_{j}^{i}\right]_{i, j-2, \ldots, n} .
$$

Comme $D, E, K$ et $L$ sont des sous-groupes fermés du groupe $H$ et comme le groupe $H$ adment les représentations:

$$
H=K \cdot G_{0} \quad \text { et } \quad H=D \cdot G_{t} \quad \text { pour } t \neq 0
$$

et

$$
H=L \cdot H_{1} \quad \text { et } \quad H=E \cdot H_{t} \quad \text { pour } t \neq 1,
$$

nous obtenons, en vertu du lemme 3, la conclusion suivante:

Conclusion 1. Le groupe $H$ n'admet pour sous-groupes fermés, contenant le sous-groupe $G_{t}$ resp. $H_{\ell}$ (pour $t$ réel fixe), que les sous-groupes de la forme: $K^{\prime} \cdot G_{0}-$ dans le cas où $t=0$ et $D^{\prime} \cdot G_{t}-$ dans le cas où $t \neq 0$ resp. $L^{\prime} \cdot H_{1}-$ dans le cas où $t=1$ et $E^{\prime} \cdot H_{t}-$ dans le cas où $t \neq 1$, où $D^{\prime}, E^{\prime}, K^{\prime}, L^{\prime}$ désignent un sous-groupe fermé arbitraire du groupe resp. $D, E, K, L$. Comme les groupes $D, E, K, L$ sont isomorphes au groupe multiplicatif des nombres réels $\boldsymbol{R}_{0}$, le théorème 1 détermine tous les sous-groupes fermés $D^{\prime}, E^{\prime}, K^{\prime}, L^{\prime}$ des groupes resp. $D, E, K, L$.

En utilisant les notations (14) nous pouvons présenter les groupes $G_{t}$ et $H_{t}$, pour $t \in R$, sous la forme suivante:

$$
\begin{aligned}
& G_{0}=\{A \in H:|a(A)|=1, d(A)>0\}, \\
& G_{t}=\left\{A \in H: d(A)=|a(A)|^{(1-t) / t}\right\} \\
& H_{1}=\{A \in H: a(A)>0,|d(A)|=1\}, \\
& H_{t}=\left\{A \in H: a(A)=|d(A)|^{t /(1-t)}\right\}
\end{aligned}
$$

Alors un calcul élémentaire montre les implications suivantes:

$$
\begin{array}{lll}
A \in H, & B \in G_{t} \Rightarrow a\left(A^{-1} B A\right)=a(B), & d\left(A^{-1} B A\right)=d(B), \\
A \in H, & B \in H_{\ell} \Rightarrow a\left(A^{-1} B A\right)=a(B), & d\left(A^{-1} B A\right)=d(B)
\end{array}
$$


pour $t_{\epsilon} \boldsymbol{R}$. Donc, pour tout $t$ réel, les groupes $G_{t}$ et $H_{t}$ sont des sous-groupes invariants du groupe $H$.

En vertu de (15) et (16), ainsi que de la commutativité des groupes: $D, E, K, L$, nous pouvons utiliser le lemme 4 et nous constatons que les sous-groupes du groupe $H$, indiqués dans la conclusion 1 , ne sont pas conjugués l'un de l'autre relativement au groupe $H$. En vertu de lemme 2 ils ne sont pas conjugués non plus relativement au groupe $G L(n)$.

En tenant compte de la conclusion 1 , du théorème 1 et du lemme 2 il en résulte les théorèmes:

THÉRÈme 2. Le groupe $G L(n)$ n'admet (sauf lui-même) pour sous-groupes fermés, contenant le suos-groupe $G_{t}$ (pour $t$ réel fixe), que les sous-groupes suivants:

$$
H, G^{+}=\{A \in H: d(A)>0\}
$$

ainsi que (dans le cas où $t=0)$ :

$$
\begin{array}{ll}
G_{0}(c)=\{A \in H: a(A) \in \boldsymbol{R}(0)\} & \text { pour } c \geqslant 1, \\
G_{0}^{*}(c)=\left\{A \in H:|a(A)|=0^{k}, \operatorname{sgn} d(A)=(-1)^{k}, k \in Z\right\} & \text { pour } c>1, \\
G_{0}^{+}(c)=\{A \in H: a(A) \in \boldsymbol{R}(c), d(A)>0\} & \text { pour } c \geqslant 1,
\end{array}
$$

et

$$
\begin{aligned}
& G_{t}(c)=\left\{A \in H:|d(A)|=c^{k}|a(A)|^{(1-t) / t}, k \in Z\right\} \quad \text { pour } c \geqslant 1, \\
& G_{i}^{*}(c)=\left\{A \in H: d(A)=(-c)^{k}|a(A)|^{(1-t) / t}, k \in Z\right\} \quad \text { pour } c>1 \text {, } \\
& G_{\ell}^{+}(c)=\left\{A \in H: d(A)=c^{k}|a(A)|^{(1-t) / \ell}, k \in Z\right\} \quad \text { pour } c \geqslant 1
\end{aligned}
$$

dans le cas oì $t \neq 0$. Les sous-groupes nommés ci-dessus ne sont pas conjugués l'un de l'autre.

THÉoRÈme 3. Le groupe $G L(n)$ n'adment (sauf lui-même) pour sous-groupes fermés, contenant le sous-groupe $H_{1}$ (pour $t$ réel fixe), que les sous-groupes suivants:

$$
H, H^{+}=\{A \in H: a(A)>0\}
$$

ainsi gue (dans le cas où $t=1$ ):

$$
\begin{array}{ll}
H_{1}(c)=\{A \in H: d(A) \in \boldsymbol{R}(c)\} & \text { pour } c \geqslant 1, \\
H_{1}^{*}(c)=\left\{A \in H:|d(A)|=c^{k}, \operatorname{sgn} a(A)=(-1)^{k}, k \in Z\right\} & \text { pour } c>1, \\
H_{1}^{+}(c)=\{A \in H: a(A)>0, d(A) \in \boldsymbol{R}(c)\} & \text { pour } c \geqslant 1,
\end{array}
$$

et

$$
\begin{array}{ll}
H_{t}(c)=\left\{A \in H:|a(A)|=c^{k}|d(A)|^{t /(1-t)}, k \in Z\right\} & \text { pour } c \geqslant 1, \\
H_{l}^{*}(c)=\left\{A \in H: a(A)=(-c)^{k}|d(A)|^{t /(1-t)}, k \in Z\right\} & \text { pour } c>1, \\
H_{\ell}^{+}(c)=\left\{A \in H: a(A)=c^{k}|d(A)|^{t /(1-t)}, k \in Z\right\} & \text { pour } c \geqslant 1
\end{array}
$$

dans le cas où $t \neq 1$. Les sous-groupes nommés ci-dessus ne sont pas conjugués l'un de l'autre. 
4. Classification des densités vectorielles factorisées. Les théorèmes 2 et 3 nous permettent, en vertu des propositions 1 et 2 ainsi que des théorèmes 1 et 2 de [6], d'établir une classification complète des factorisations (comitants) des densités vectorielles. Nous la présenterons dans les conclusions suivantes:

Conclusion 2. Toute factorisations (comitant) de la densité vectorielle ordinaire $V_{t}$ de poids $-t$ est équivalente à l'un des objets factorisés suivants: $\Sigma=V_{t} / G L(n)$ (scalaire trivial), $\Delta=V_{t} / H$ (direction), $\Delta^{*}=V_{t} / G^{+}$, $\nabla_{t}(c)=V_{t} / G_{t}(c)$ pour $c \geqslant 1, \quad V_{t}^{*}(c)=V_{t} / G_{i}^{*}(c)$ pour $c>1$ et $V_{t}^{+}(c)=$ $=V_{t} G_{t}^{*}(c)$ pour $c \geqslant 1$. Les objets géométriques nommés ci-dessus ne sont pas équivalents l'un à l'autre.

Conclusion 3. Toute factorisation (comitant) de la densité vectorielle du type de $H$. Weyl $W_{t}$ de poids $-t$ est équivalente à l'un des objets factorisés suivants: $\Sigma=W_{t} / G L(n)$ (scalaire trivial), $\Delta=W_{t} / H$ (direction), $\Delta^{+}=W_{t} / H^{+}$(direction munie de sens), $W_{t}(c)=W_{t} / H_{t}(c)$ pour $c \geqslant 1$, $W_{t}^{*}(c)=W_{t} / H_{t}^{*}(c)$ pour $c>1, W_{t}^{+}(c)=W_{t} / H_{t}^{+}(c)$ pour $c \geqslant 1$. Les objets géométriques nommés ci-dessus ne sont pas équivalents l'un à l'autre.

En utilisant notre proposition 3 ainsi que les inclusions entre les sous-groupes du groupe $G L(n)$ déterminés dans les théorèmes 2 et 3 , nous pouvons donner une classification des factorisations (comitants) des objets factorisés mentiounés dans les conclusions 2 et 3 . Nous présenterons cette classification dans conclusion suivante:

Conclusion 4. Les densités vectorielles factorisées n'admettent (sauf elles-mêmes et le scalaire trivial $\Sigma$ ) de factorisations (comitants) que celles qui sont équivalentes aux objets nommés dans la suite:

$\Sigma$ et $\Delta-$ n'admettent aucune factorisation non triviale,

$\Delta^{*}$ et $\Delta^{+}$- direction $\Delta$,

$V_{t}(c)-V_{t}\left(c^{1 / p}\right)$ pour $p=2,3, \ldots$ et $\Delta$,

$W_{t}(c)-W_{t}\left(c^{1 / p}\right)$ pour $p=2,3, \ldots$ et $\Delta$,

$V_{t}^{*}(c)-V_{t}^{*}\left(c^{1 / 2 p-1}\right)$ pour $p=2,3, \ldots, V_{t}\left(c^{1 / p}\right)$ pour $p=1,2, \ldots$ et $\Delta$, et $\Delta$,

$W_{t}^{*}(c)-W_{t}^{*}\left(c^{1 / 2 p-1}\right)$ pour $p=2,3, \ldots, W_{t}\left(c^{1 / p}\right)$ pour $p=1,2, \ldots$

$V_{t}^{+}(c)-V_{t}^{+}\left(c^{1 / p}\right)$ pour $p=2,3, \ldots, V_{i}^{*}\left(c^{1 / 2 p}\right)$ pour $p=1,2, \ldots$ $\nabla_{t}\left(c^{1 / p}\right)$ pour $p=1,2, \ldots, \Delta^{*}$ et $\Delta$,

$W_{l}^{+}(c)-W_{t}^{+}\left(c^{1 / p}\right)$ pour $p=2,3, \ldots, W_{t}^{*}\left(c^{1 / 2 p}\right)$ pour $p=1,2, \ldots$, $W_{t}\left(c^{1 / p}\right)$ pour $p=1,2, \ldots, \Delta^{+}$et $\Delta$.

Cette classification est vraie pour tout $c>1$ et $t \in \boldsymbol{R}$.

Remarque. Comme la densité vectorielle $W_{0}$ se réduit au vecteur contravariant (envisagé comme un objet géométrique abstrait) la conclusion 3 établit une classification des factorisations du vecteur contravariant. 
Le sens géométrique des factorisations $\Delta$ et $\Delta^{+}$est évident, tandis que le sens des autres factorisations exige encore certaines explications, même dans le cas du vecteur contravariant $W_{0}$.

\section{Travaux cités}

[1] Е. Ђ. Дынкин, Максимальные подерупы классических ерупп, Труды Моск. Мат. Обц. I (1952), p. 39-166.

[2] J. Haantjes and G. Laman, On the definition of geometric objects I, II, Nederl. Akad. Wetensch. Proc. ser. A 56 = Indagationes Math. 15 (1953), p. 208-215 and 216-222.

[3] M. Kucharzewski and M. Kuczma, Basic concepts of the theory of geometric objects, Rozprawy Matematyczne 43, Państwowe Wydawnictwo Naukowe, WarBzawa 1964.

[4] M. Kucharzewski und A. Zajtz, Klassifikation der linearen homogenen geometrisohen Objekte deren Komponentenzahl die Dimension des Raumes nicht übertrifft, Colloq. Math. 16 (1967), p. 185-192.

[5] E. Siwek, Sur les comitants algébriques des objets géométriques non transitifs, Ann. Polon. Math. 24 (1970), p. 27-33.

[6] - et A. Zajtz, Contribution à la théorie des pseudoobjets géométriques, ibidem 19 (1967), p. 185-192.

[7] - - Sur les comitants algébriques des densités, Prace Naukowe Uniwersytetu. Ślaskiego nr 2, Prace Matematyczne I (1969), p. 91-98.

Reçu par la Rédaction le 5. 1. 1974 